\title{
A ENFERMAGEM DE REABILITAÇÃO NO EMPODERAMENTO E CAPACITAÇÃO DA PESSOA EM PROCESSOS DE TRANSIÇÃO SAÚDE-DOENÇA
}

\section{ENFERMERÍA DE REHABILITACIÓN EN EL EMPODERAMIENTO Y CAPACITACIÓN DE PERSONAS EN PROCESOS DE TRANSICIÓN SALUD-ENFERMEDAD}

\section{REHABILITATION NURSING IN PERSON'S EMPOWERMENT AND TRAINING IN HEALTH-DISEASE TRANSITION PROCESSES}

\author{
DOI 10.33194/rper.2020.v3.n1.8.5763 | Submetido 28/02/2020 | Aprovado 08/08/2020
}

\section{Luís Manuel Mota de Sousa' ${ }^{1}$; ; Maria Manuela Martins² ${ }^{2}$; André Novo $^{3}$ (D)}

1 - Escola Superior de Enfermagem S. João de Deus, Comprehensive Health Research Centre Universidade de Évora; 2 - Escola Superior de Enfermagem do Porto; 3 - Instituto Politécnico de Bragança, Escola Superior de Saúde

\section{RESUMO}

Objetivo: Discutir a contribuição da Enfermagem de Reabilitação para o Empoderamento e a Capacitação da pessoa em processos de transição saúde-doença.

Método: Prática reflexiva com discussão norteada pela Teoria das Transições de Afaf Ibrahim Meleis, os diagnósticos de enfermagem segundo a linguagem da Classificação Internacional para a Prática de Enfermagem e as competências prescritas para o enfermeiro de reabilitação.

Resultados: Os enfermeiros de reabilitação desenvolvem um processo de capacitação privilegiando o foco de atenção o conhecimento e aprendizagem de capacidades desenvolvendo intervenções de modo a contribuir para indicadores específicos dos processos de transição saúde doença. Estes resultados assentam em conceitos subsidiários de boas práticas preconizadas com o objetivo último de uniformizar linguagem e terminologia dos enfermeiros de reabilitação.

Conclusão: A intervenção do enfermeiro de reabilitação nos focos conhecimento e aprendizagem de capacidades da pessoa permite o empoderamento, a tomada de decisão e a passagem à ação, de modo a desenvolver habilidades e assim fazer a pessoa sentir-se capacitada para lidar com os desafios que surgem no dia a dia, decorrentes de processos de transição.

Descritores: Teoria de Enfermagem; Transições; Enfermagem de Reabilitação; Capacitação; Empoderamento para a Saúde

\section{RESUMEN}

Objetivo: Discutir la contribución de la Enfermería de Rehabilitación al Empoderamiento y Capacitación de la persona en los procesos de transición salud-enfermedad.

Método: Práctica reflexiva con discusión guiada por la teoría de las transiciones de Afaf Ibrahim Meleis, diagnósticos de enfermería según el lenguaje de la Clasificación Internacional para la Práctica de Enfermería y las habilidades prescritas para la enfermera de rehabilitación.

Resultados: Los enfermeros de rehabilitación desarrollan un proceso de capacitación centrado en el enfoque de atención en el conocimiento y las habilidades de aprendizaje, desarrollando intervenciones de manera a contribuir para los indicadores específicos de los procesos de transición de salud-enfermedad. Estos resultados se basan en conceptos que respaldan las buenas prácticas defendidas con el objetivo final de estandarizar el lenguaje y la terminología de los enfermeros de rehabilitación.

Conclusión: La intervención del enfermero de rehabilitación en el enfoque del conocimiento y aprendizaje de las habilidades de la persona permite el empoderamiento, la toma de decisiones y la transición a la acción, a fin de desarrollar habilidades y, por lo tanto, sentirse empoderado para enfrentar los desafíos que surgen en el día a día, derivado de procesos de transición.

Descriptores: Teoría de Enfermería; Transiciones; Enfermería en rehabilitación; Capacitación; Empoderamiento para la salud

\section{ABSTRACT}

Objective: To discuss the contribution of Rehabilitation Nursing to the Empowerment and Training of the person in health-disease transition processes.

Method: Reflective practice with discussion guided by the Afaf Ibrahim Meleis Theory of Transitions and by the International Classification for Nursing Practice diagnoses and the skills prescribed for the rehabilitation nurse. 
Results: The qualification process of rehabilitation nurses' privileges knowledge and learning skills, allowing the development of interventions that contribute to specific indicators of the health-disease transition processes. These results are based on concepts that support good practices that aim to standardize the language and terminology of rehabilitation nurses.

Conclusion: The intervention of the rehabilitation nurse in the focus of knowledge and learning of person's abilities allows empowerment, decision making and transition to action. It also enables the person to develop skills and feel empowered to deal with the daily challenges that arise from transition processes.

Descriptors: Nursing Theory; Transitions; Rehabilitation Nursing; Training; Empowerment for Health

\section{INTRODUÇÃO}

A educação da pessoa, no âmbito da saúde, na perspetiva crítica, deve ser vista como processo, em que aprender implica construir e não adquirir conhecimentos; significa desenvolver habilidades pessoais e sociais e não adaptar ou reproduzir comportamentos ${ }^{(1)}$.

$\mathrm{Na}$ educação para a saúde tem-se dado um novo enfoque nas práticas educativas, de modo a fortalecer a capacidade de escolha das pessoas, ou seja, tem de permitir que estas participem ativamente no processo de tomada de decisão e de implementação de estratégias para a melhoria das suas condições de saúde ${ }^{(2)}$.

Capacitar, no contexto da educação para a saúde, é um processo multidimensional que envolve conhecimento, decisão e ação. Os conhecimentos são os saberes construídos e alicerçados nos valores da pessoa, que se modificam ao longo da vida e são influenciados por fatores, nomeadamente de ordem social, cultural e religiosa ${ }^{(3)}$.

O empoderamento está relacionado com o conhecimento e a autonomia na tomada de decisão. A autonomia define-se com a liberdade da pessoa em gerir livremente a sua vida efetuando, racionalmente, as suas próprias escolhas ${ }^{(4)}$. Neste sentido, o processo de empoderamento tem como fim a emancipação da pessoa, mediante a promoção da autonomia e alcance de medidas terapêuticas disponíveis, independentemente dos recursos didáticos e avaliativos disponíveis(5).

Além disso, a (re)construção do conhecimento condiciona a decisão sobre a ação a qual se altera no decurso do tempo, sendo o resultado de um conjunto de fatores como a decisão tomada, a sua capacidade (limitação da atividade) e os recursos disponíveis para a executar. Nesta perspetiva, a capacitação é um processo que envolve os domínios cognitivo, físico e material ${ }^{(3)}$.

Importa então refletir como os enfermeiros contribuem especificamente para o empoderamento e capacitação a partir do conhecimento desta área do saber. De facto, o pensamento teórico em enfermagem evoluiu e, continua a evoluir num percurso de significativa riqueza concetual, que deveria ser apropriada pelos enfermeiros, ${ }^{(6)}$ pelo que faz sentido compreender estes dois conceitos suportados num referencial teórico.

Procurar significado teórico para a intervenção dos enfermeiros, é relembrar que a disciplina de enfermagem está relacionada com as experiências humanas de transição, nas quais a saúde e o bem-estar podem ser considerados resultados da sua intervenção. A pessoa sofre descontinuidades no processo de vida em que a doença pode ser um dos motivos. Esta mudança consciente e paulatinamente integrada no íntimo da pessoa vai provocar alterações que levam a que pessoa enfrente a nova situação de forma mais saudável.

Nesse sentido, o desafio para os enfermeiros é entender os processos de transição e desenvolver terapêuticas efetivas que ajudem as pessoas a recuperar a estabilidade e 0 bem-estar ${ }^{(7-8)}$ logo a ficarem empoderadas e capacitadas para fazer face à continuidade da sua vida.. Assim, quando se realiza a capacitação de um cuidador familiar, faz-se porque este desempenha um conjunto de tarefas no quotidiano que necessitam de conhecimento e treino, tais como atividades de vida diárias, resolução de problemas, tomada de decisão, atividades que requerem competências comunicativas e organizacionais, assim como cuidados antecipatórios e de vigilância ${ }^{(9)}$.

Os processos de capacitação são adaptações que podem ocorrer de forma progressiva, em associação com as alterações que ocorrem ao longo do ciclo vital ou, então, bruscamente, causadas por intercorrências vivenciais ${ }^{(3)}$ além disso, tem de se ter em consideração que pessoa vive distintas transições em simultâneo e que tem o direito a conhece-las para tomar decisões de forma autónoma.

\section{OBJETIVO}

Discutir a contribuição da Enfermagem de Reabilitação para o Empoderamento e a Capacitação da pessoa em processos de transição saúde-doença.

\section{MÉTODO}

O método utilizado para o desenvolvimento do presente artigo foi a prática reflexiva. Para tal, começou-se por selecionar o tema a abordar; após esta seleção procedeu-se à discussão entre os autores, à escolha dos artigos que serviram de base para o presente artigo e que permitiram a discussão reflexiva do tema. Esta discussão reflexiva teve como base a Teoria das Transições de Afaf Ibrahim Meleis ${ }^{(7-8)}$ e os diagnósticos de enfermagem segundo a linguagem da Classificação Internacional para a Prática de Enfermagem $(\mathrm{CIPE})^{\left({ }^{(10)} \mathrm{e}\right.}$ as competências específicas do enfermeiro de reabilitação definidas pela Ordem dos Enfermeiros ${ }^{(11)}$. 


\section{RESULTADOS}

Os resultados do presente artigo são apresentados mediante discussão reflexiva sobre a contribuição da Enfermagem de Reabilitação para o Empoderamento e a Capacitação da pessoa em processos de transição saúde doença. Os Enfermeiros de reabilitação desenvolvem um processo de capacitação privilegiando o foco de atenção o conhecimento e aprendizagem de capacidades, desenvolvendo intervenções no sentido contribuir para indicadores específicos deste processo de transição.

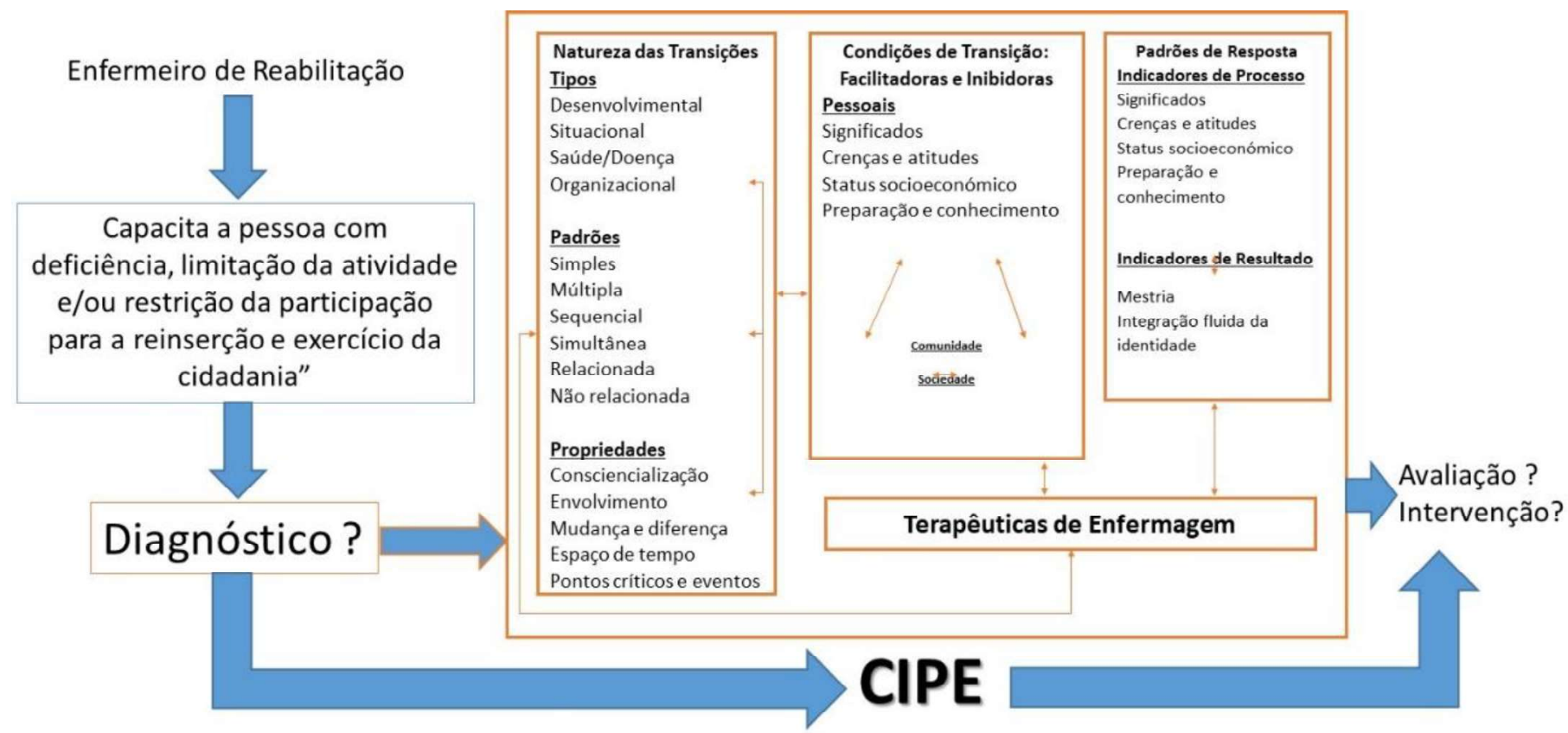

Figura 1 - Resultados identificados para a análise, adaptado de Meleis(8)

Estes resultados assentam em conceitos subsidiadores de boas práticas preconizadas com a finalidade de contribuir para a uniformização da linguagem e da terminologia dos enfermeiros de reabilitação.

\section{DISCUSSÃO}

A teoria de médio alcance sobre as transições é constituída pela natureza das transições (tipos, padrões e propriedades); condicionantes facilitadores e inibidores da transição (pessoais, comunidade e sociedade); padrões de resposta (indicadores de processos e indicadores de resultados) e terapêutica de enfermagem. As transições resultam das mudanças na vida, na saúde, nos relacionamentos e nos ambientes. Este processo de transição é caraterizado pela sua singularidade, diversidade, complexidade e múltiplas dimensões que geram significados variados e são determinados pela perceção de cada indivíduo ${ }^{(7-8)}$.

Considerando a teoria de Meleis ${ }^{(8)}$, a transição é uma passagem de um estado estável para outro estado estável a partir de um processo que emerge a partir de uma mudança, por isso as transições tornam-se dinâmicas mas também marcos de viragem, podendo ser caraterizadas a partir deste processo ou de resultados finais.

A consciencialização está relacionada com a perceção, o conhecimento e o reconhecimento de uma experiência de transição. É uma característica definidora de transição, cuja ausência significa que a pessoa pode não ter iniciado a experiência de transição.
A pessoa só pode envolver-se depois de se consciencializar das mudanças físicas, emocionais, sociais ou ambientais ${ }^{(7)}$.

Os indicadores de processo referem-se: sentir-se ligado às redes de apoio como família/amigos/profissionais de saúde; o interagir com pessoas que estão na mesma situação, profissionais de saúde, cuidadores familiares de modo a clarificar e ajustar os comportamentos de resposta às transições; o estar situado no tempo, espaço e relações, pois possibilita que a pessoa se desprenda de seu passado e enfrente novos desafios; e o desenvolver confiança e ajustamento, que se manifesta pelo nível de compreensão dos diferentes processos relativos à necessidade de mudança, utilização de recursos e desenvolvimento de estratégias para ganhar confiança e lidar com a situação ${ }^{(7)}$.

Os indicadores de resultado estão relacionados com mestria, ou seja, ao domínio de novas competências; e à integração fluida de identidade que se refere à reformulação da identidade, de modo a ficar mais fluida e dinâmica. Nesta ótica, a capacidade ou habilidade para desenvolver novas competências é fundamental para fazer a transição com sucesso ${ }^{(7)}$.

As terapêuticas de enfermagem e os indicadores de processo e resultado podem ser observados nas competências especificas do enfermeiro de reabilitação no âmbito da capacitação da pessoa para lidar a transição em que se encontra e respetivos ensinos e treinos que são realizados pelo enfermeiro de reabilitação.

A competência específica do enfermeiro de reabilitação enuncia que este “J2 - Capacita a pessoa com 
deficiência, limitação da atividade e/ou restrição da participação para a reinserção e exercício da cidadania" e apresenta a unidade de competência “J2.1

- Elabora e implementa programa de treino de atividades de vida diária visando a adaptação às limitações da mobilidade e à maximização da autonomia e da qualidade de vida." Com os seguintes critérios de avaliação “J2.1.1 - Ensina a pessoa e/ou cuidador técnicas e tecnologias específicas de autocuidado; J2.1.2 - Realiza treinos específicos de atividades de vida diária, nomeadamente utilizando produtos de apoio (ajudas técnicas e dispositivos de compensação), assim como os treinos inerentes à atividade e exercício físico; e J2.1.3 - Ensina e supervisa a utilização de produtos de apoio (ajudas técnicas e dispositivos de compensação) tendo em vista a máxima capacidade funcional da pessoa"(11).

No processo de capacitação da pessoa com deficiência, limitação da atividade e restrição da participação, o enfermeiro especialista em enfermagem de reabilitação tem dois focos de atenção - o "conhecimento" e a "aprendizagem de capacidades". Vai utilizar o conhecimento para empoderar a pessoa para a tomada de decisão de modo a maximizar a sua autonomia, por um lado, e por outro vai servir-se da aprendizagem de capacidades para tornar a pessoa mais independente na realização das atividades de vida diária.

É ainda de salientar que o processo de capacitação não se limita à pessoa enquanto individualidade singular, mas também o seu contexto como seja a família, o ambiente de trabalho e social, porque os conhecimentos e as aprendizagens não se limitam às pessoas com deficiência.

0 enfermeiro de reabilitação tendo como foco de atenção o conhecimento, prescreve intervenções como "Ensinar sobre (...)", "Orientar para o uso de (...)" de modo a obter ganhos em conhecimento, permitindo assim melhorar a tomada de decisão e autonomia. Contribui, deste modo para a obtenção de indicadores de processo de uma transição bem-sucedida, nomeadamente sentir-se ligado, interagir, estar situado, assim como desenvolver confiança e ajustamento. Além disso, existem outros indicadores relacionado com o foco conhecimento que podem ser analisados do ponto de vista de estrutura, processo e resultado (Figura 1 e Quadro 1).

A aprendizagem de capacidades implica passar para ação o que se adquiriu como o empoderamento e tomada de decisão, de modo a tornar a pessoa mais independente na execução das atividades básicas e instrumentais de vida diária, contribuindo assim para uma transição com sucesso, verificada pela perceção de mestria na execução dessas atividades e integração de uma nova identidade (consciencialização de que é capaz e está diferente da situação anterior). Associado a estes indicadores de resultado de transição saudável e bem-sucedida, os enfermeiros com as intervenções "instruir sobre (...)", "treinar a técnica de (...)" e "treinar o uso de (...)" podem medir a efetividades destas a nível dos ganhos em aprendizagem de capacidades através de indicadores de estrutura, processo e resultado (Figura 1 e Quadro 1).
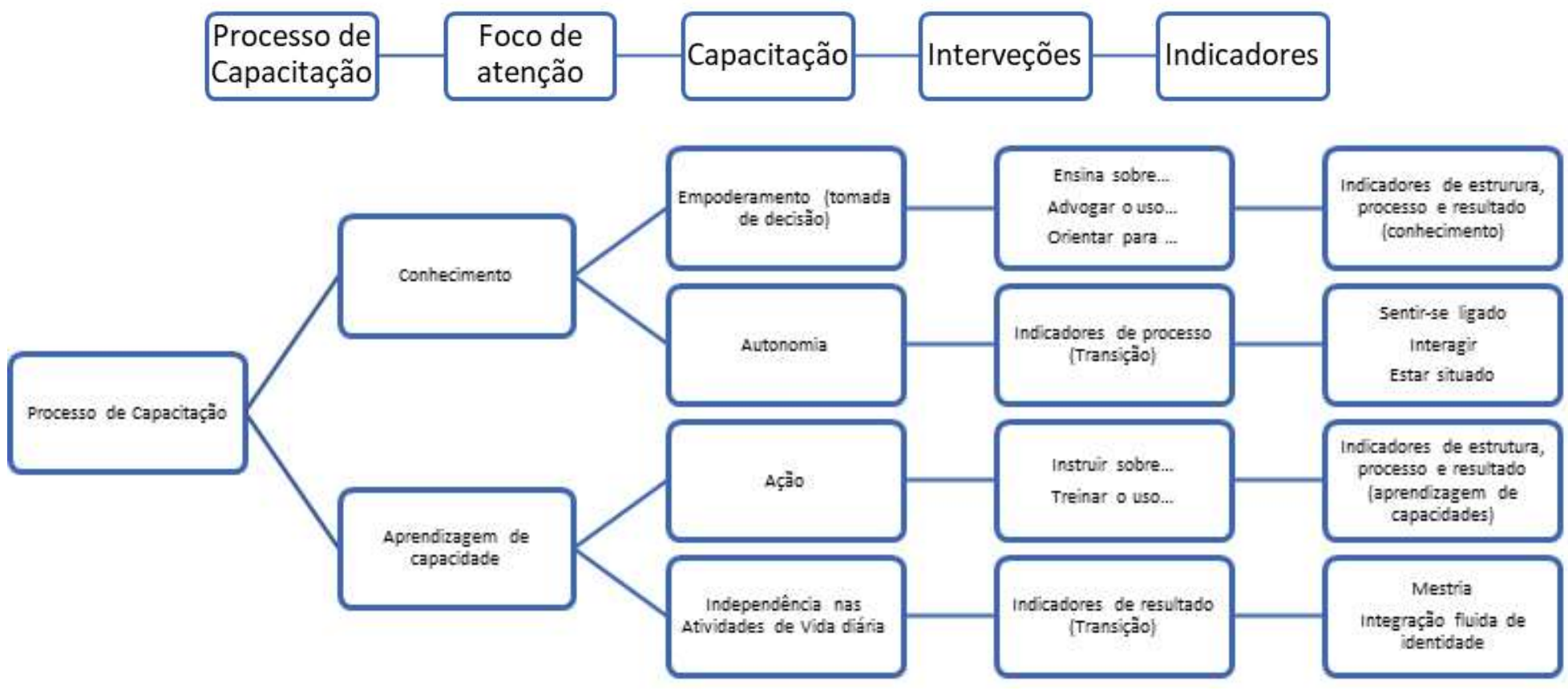

Figura 2 - Capacitação da pessoa em processos de transição 


\section{Foco/Diagnóstico de enfermagem}

Intervenções de enfermagem

\section{Indicadores}

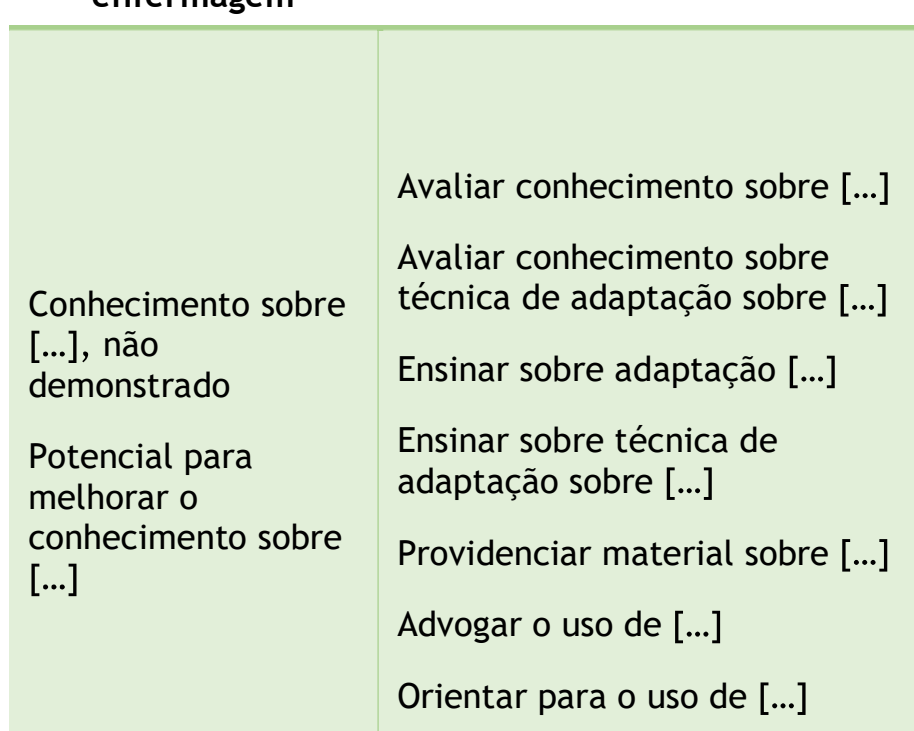

Aprendizagem de capacidades sobre [...], não demonstrada

Potencial para melhorar a aprendizagem de capacidades [...]

\section{Avaliar capacidade para [...]}

Instruir técnica de adaptação [...]

Instruir sobre o uso de dispositivo [...]

Treinar técnica de adaptação[...]

Treinar o uso de dispositivo [...]
Estrutura - Percentagem de material educativo sobre adaptação [...] disponibilizado aos clientes que acompanham (Número de dipositivos de educação sobre as técnicas de adaptação [...] face ao número de clientes seguidos)

Processo - Percentagem de clientes com potencial para melhorar conhecimento sobre técnica de adaptação [...] (Número de clientes com potencial de melhorar os conhecimentos sobre as técnicas de adaptação [...] face ao número de clientes seguidos)

Resultado - Ganhos em conhecimento sobre adaptação [...] (Número de clientes que demonstraram os conhecimentos sobre a adaptação [...] face ao número de clientes seguidos)

Resultado - Ganhos em conhecimento sobre técnica de adaptação [...] (Número de clientes que demonstraram os conhecimentos sobre técnicas de adaptação [...] face ao número de cientes seguidos)

Processo - Percentagem de clientes com potencial para melhorar capacidade [...] (Número de clientes com potencial de melhorar a aprendizagem sobre as técnicas de adaptação [...] face ao número de clientes seguidos)

Resultado - Ganhos em capacidade para [...] (Número de clientes que demonstraram aprendizagem de capacidade sobre técnicas de adaptação [...] face ao número de cientes seguidos)

Quadro 1 - Diagnósticos, resultados, intervenções e indicadores de enfermagem nos focos conhecimento e aprendizagem de capacidade

\section{IMPLICAÇÕES PRÁTICAS}

Os diagnósticos de enfermagem do foco conhecimento e aprendizagem de capacidades podem ser alvo de intervenção dos enfermeiros de reabilitação, uma vez que as competência “J2 - Capacita a pessoa com deficiência, limitação da atividade e/ou restrição da participação para a reinserção e exercício da cidadania" e "J3 - Maximiza a funcionalidade desenvolvendo as capacidades da pessoa"(11) os habilitam para o desenvolvimento e implementação de ações autónomas a nível do ensino, instrução e treino (Quadro 1) para capacitar a pessoa na realização de atividades básicas e instrumentais de vida diária e para melhorar os processos de transição e a qualidade de vida.

\section{IMPLICAÇÕES PRÁTICAS}

Os diagnósticos de enfermagem do foco conhecimento e aprendizagem de capacidades podem ser alvo de intervenção dos enfermeiros de reabilitação, uma vez que as competência “J2 - Capacita a pessoa com deficiência, limitação da atividade e/ou restrição da participação para a reinserção e exercício da cidadania" e "J3 - Maximiza a funcionalidade desenvolvendo as capacidades da pessoa"(11) os habilitam para o desenvolvimento e implementação de ações autónomas a nível do ensino, instrução e treino (Quadro 1) para capacitar a pessoa na realização de atividades básicas e instrumentais de vida diária e para melhorar os processos de transição e a qualidade de vida.

\section{CONSIDERAÇÕES FINAIS}

O enfermeiro de reabilitação tem como competência capacitar a pessoa com deficiência, limitação da atividade e/ou restrição da participação, de modo a maximizar a autonomia, facilitar a realização das atividades básicas e instrumentais de vida diária, melhorar a participação na sociedade e consequentemente a perceção da qualidade de vida relacionada com a saúde, tomando particular ênfase no processo de transição saúde-doença.

A intervenção nos focos conhecimento e aprendizagem de capacidades da pessoa permite o empoderamento, a tomada de decisão e a passagem à ação, de modo a desenvolver habilidades e assim sentir-se capacitada para lidar com os desafios que surgem no dia a dia, decorrentes de processos de transição. 
Pode-se afirmar que o foco de atenção conhecimento e aprendizagem desenvolvida pelos enfermeiros de reabilitação transcende a intervenção do cliente enquanto pessoa, mas também, se desenvolve no sentido do cliente família e comunidades.

Com esta reflexão acreditamos que fica sustentada a intervenção dos enfermeiros de reabilitação com a finalidade de proporcionar Capacitação à pessoa com deficiência, limitação da atividade e/ou restrição da participação, contudo acreditamos que o modelo não traz contributos suficientes para a intervenção, afim de fazer face à reinserção e exercício da cidadania em plena por isso era importante trabalhar o sucesso das pessoas após as transições efetuadas. Esta limitação deixa em aberto a necessidade de continuar a refletir e a investigar as transições bem-sucedidas.

\section{REFERÊNCIAS BIBLIOGRÁFICAS}

1. Lopes R, Tocantis, FR. Promoção da saúde e a educação crítica. Interface Comun Saúde Educ. 2012;16(40): 235-46. Disponível em https: / /www.scielosp.org/article/icse/2012.v16n40/235-248/

2. Costa DW, Parreira BD, Borges FA, dos Santos DM, Tavares LD, Goulart BF. Educação em saúde e empoderamento do usuário da estratégia saúde da família. Rev enferm UFPE online. Recife. 2016 Jan;10(1):96-102. Disponível em: https://doi.org/10.5205/reuol.8423-73529-1-RV1001201613

3. Reis G, Bule MJ. Capacitação e Atividade de Vida. In C. MarquesVieira, L. Sousa (Eds). Cuidados de Enfermagem de Reabilitação à Pessoa ao Longo da Vida. Loures: Lusodidacta; 2016:57-66.

4. Dutra VF, Bossato HR, Oliveira RM. Mediar a autonomia: um cuidado essencial em saúde mental. Escola Anna Nery. 2017;21(3): e20160284. Disponível em: https://doi.org/10.1590/2177-9465-ean2016-0284

5. Vale PL; Prata DRA, , Araújo CO, Cordeiro MB, Goés ÂC. Estratégias de empoderamento em saúde: dialogando o plano municipal e o relatório da conferência de saúde. Rev Electrón Gest Saúde. 2018;9(1):78-94. Disponível em: http://periodicos.unb.br/index.php/rgs/article/download/10431/9 189

6. Queirós PJ. O conhecimento em enfermagem e a natureza dos seus saberes. Esc Anna Nery Rev Enferm, 2016;20(3): e201600/9. Disponível em: https://doi.org/10.5935/1414-8145.20160079

7. Meleis Al, Sawyer LM, Im EO, et al. Experiencing Transitions: An Emerging MiddleRange Theory. Adv Nurs Sci. 2000; 23(1): 12-28. Disponível em: https://doi.org/10.1097/00012272-200009000-00006

8. Meleis Al. Theoretical nursing: Development and progress. 5. Ed. Philadelphia: Lippincott Williams \& Wilkins; 2011.

9. Sousa L, Sequeira C, Ferré-Grau C, Neves P, Lleixà-Fortuño M. Características de um programa de capacitação para familiares cuidadores de pessoas com demência a residir no domicílio. Rev Port Enferm Saúde Mental. 2016 Apr(SPE3):33-8. Disponível em: http://dx.doi.org/10.19131/rpesm.0114

10. Conselho Internacional de Enfermeiros. CIPE $®$ Versão 2015 classificação internacional para a prática de enfermagem. Lisboa: Ordem dos Enfermeiros; 2016. Disponível em: https: / / futurosenf. files.wordpress.com/2017/04/cipe_2015.pdf

11. Ordem dos enfermeiros. Regulamento das competências específicas do enfermeiro especialista em enfermagem de reabilitação. Lisboa: Ordem dos enfermeiros. 2019. (Regulamento n. ${ }^{\circ}$ 392/2019. Diário da República, 2. ${ }^{\mathrm{a}}$ série $-\mathrm{N} .{ }^{\circ} 85-3$ de maio de 2019. Páginas 13565-68). Disponível em: https://dre.pt/application/conteudo/122216893

12. Ordem dos Enfermeiros. Padrão Documental dos Cuidados de Enfermagem da Especialidade de Enfermagem de Reabilitação. Lisboa: Ordem dos enfermeiros. Mesa do Colégio da Especialidade de Enfermagem de Reabilitação. 2015. Disponível em: https://www.ordemenfermeiros.pt/arquivo/colegios/Documents/20 15/MCEER_Assembleia/PadraoDocumental_EER.pdf

13. Ordem dos Enfermeiros. Bilhetes de identidade dos indicadores que integram o core de indicadores por categoria de enunciados descritivos dos padrões de qualidade dos cuidados de enfermagem de reabilitação. Lisboa: Ordem dos Enfermeiros. 2018. Disponível em https://www.ordemenfermeiros.pt/media/5443/enfermagemreabili tacao.pdf 